\title{
Frecuencia de helmintos gastrointestinales en roedores sinantrópicos capturados en el Zoológico de Barranquilla
}

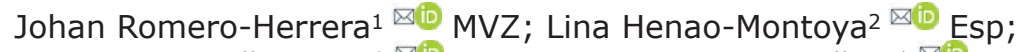 \\ Henrique Guimarães-Riva2 ${ }^{\square(0)} M V$; Jeiczon Jaimes Dueñez ${ }^{1 *} \bowtie(0)$ Ph.D.
}

\begin{abstract}
${ }^{1}$ Universidad Cooperativa de Colombia, Facultad de Medicina Veterinaria y Zootecnia. Grupo de Investigación en Ciencias Animales - GRICA, Calle 30 No. 33-51, Bucaramanga, Colombia.

²Fundación Botánica y Zoológica de Barranquilla (FUNDAZOO). Departamento de Veterinaria, Barranquilla, Colombia.

*Correspondencia: jeiczon05@gmail.com
\end{abstract}

Recibido: Marzo 2020; Aceptado: Junio 2020; Publicado: Agosto 2020.

\section{RESUMEN}

Objetivo. El objetivo del presente trabajo fue describir la frecuencia y caracterización de parásitos gastrointestinales en roedores sinantrópicos capturados en el Zoológico de Barranquilla. Materiales y métodos. Para la captura de los roedores se utilizaron trampas Tomahawk con ayuda de cebos no tóxicos, y posteriormente los individuos capturados fueron sacrificados mediante la inhalación de isoflurano. El diagnóstico e identificación de parásitos en el contenido intestinal fue realizado mediante las técnicas de examen directo en $\mathrm{NaCl}(0.9 \%)$, flotación Sheather modificada y sedimentación Ritchie modificada. Resultados. Un total de 34 roedores (Rattus rattus) fueron capturados, en los cuales la frecuencia de infección fue de $82.4 \%$ (IC 95\% = 68.8-95.8\%, 28/34). Los parásitos descritos con mayor frecuencia fueron los nematodos $(63.2 \%$, IC $95 \%=49.2-77.2 \%, 31 / 49)$, seguido de los protozoos $(20.4 \%$, IC $95 \%=8.7-32.1 \%, 10 / 49)$ y cestodos $(16.3 \%$, IC $95 \%=5.6-27 \%, 8 / 49)$, con diferencias significativas entre los tres grupos $\left(x^{2}=20.7, p<0.01\right)$. Dentro de los nematodos, la familia encontrada con mayor frecuencia fue la Strongylidae (24/31), seguida de la Ascarididae (4/31), Ancylostomatidae (1/31), Oxyuridae (1/31) y Trichostrongylidae (1/31); para el caso de los protozoos, fueron la Eimeriidae (6/10) y Entamoebidae (4/10), mientras para los cestodos fue la Hymenolepididae (8/8). Conclusiones. La especie $R$. rattus fue la única especie de roedor sinantrópico capturada en el Zoológico de Barranquilla, la cual al encontrarce infectada con diversas especies de parásitos gastrointestinales, debe ser considerada un factor de riesgo para la transmisión de estos parásitos hacia personas y otros animales en el zoológico.

Palabras clave: Infección; epidemiología; parasitología; mamíferos (Fuente: DeCS).

\section{ABSTRACT}

Objective. The aim of this work was to characterize and describe the frequency of the gastrointestinal parasites in synanthropic rodents captured at the Barranquilla Zoo. Materials and methods. The rodents were captured using Tomahawk traps with the help of non-toxic bait and subsequently 
sacrificed by isoflurane inhalation. The diagnosis and identification of parasites in the intestinal contents was performed using direct examination in $\mathrm{NaCl}(0.9 \%)$, modified Sheather's flotation, and modified Ritchie's sedimentation techniques. Results. A total of 34 rodents (Rattus rattus) were captured, with an infection frequency of $82.4 \%(95 \% \mathrm{CI}=68.8 \%-95.8 \%, 28 / 34)$. The most prevalent parasites were nematodes $(63.2 \%, 95 \% \mathrm{CI}=49.2 \%-77.2 \%, 31 / 49)$, followed by protozoans $(20.4 \%, 95 \%$ $\mathrm{CI}=8.7 \%-32.1 \%, 10 / 49)$ and cestodes $(16.3 \%, 95 \% \mathrm{CI}=5.6 \%-27 \%, 8 / 49)$, with significant differences between the three groups $\left(X^{2}=20.7, p<0.01\right)$. Among the nematodes, Strongylidae was the most prevalent family (24/31), followed by Ascarididae (4/31), Ancylostomatidae (1/31), Oxyuridae (1/31), and Trichostrongylidae (1/31); for the protozoans, the most prevalent families were Eimeriidae (6/10) and Entamoebidae (4/10), and for the cestodes, the only family found was the Hymenolepididae (8/8) family. Conclusions. The $R$. rattus species was the only species of synanthropic rodents caught in the Barranquilla Zoo; because it was infected with various species of gastrointestinal parasites, it should be considered a risk factor for the transmission of these parasites to humans and other animals in the zoo.

Keywords: Infection; epidemiology; parasitology; mammals (Source: DeCS).

\section{INTRODUCCIÓN}

Los roedores sinantrópicos constituyen especies dominantes, encontrándose en la mayoría de las regiones del mundo debido a su gran capacidad de adaptación (1). Así mismo, revisten de importancia en la cadena epidemiológica, pues son claves en la transmisión de diversos parásitos en animales silvestres y domésticos, incluido los seres humanos (1). Entre los agentes etiológicos transmitidos por roedores se encuentran varias especies de bacterias: Leptospira interrogans, L. kirschneri, Borrelia burgdorferi, Rickettsia sp., Bartonella sp.; protozoos: Entamoeba coli, E. muris, Trypanosoma cruzi, T. lewisi, Chilomastix intestinalis; nematodos: Calodium hepaticum, Syphacia sp., Trichuris sp. Strongyloides sp., y cestodos: Hymenolepis diminuta, H. nana, Taenia sp., entre otros $(2,3)$. La mayoría de estos agentes infecciosos se transmiten por contacto con heces y orina de roedores infectados y/o a través de sus ectoparásitos como Xenopsilla cheopis y Nosopsyllus fasciatus $(2,3)$.

En Colombia, aunque los roedores han demostrado ser importantes en la trasmisión de microorganismos zoonóticos como $T$. cruzi, Leptospira sp., Rickettsia sp., Toxoplasma gondii, entre otros $(4,5,6,7)$, los estudios con relación a la fauna parasitaria de estos animales y su rol como posibles transmisores de parásitos gastrointestinales con impacto en la salud pública y animal, son escasos $(8,9)$. Algunos estudios con relación a esta temática sugieren una mayor positividad de parásitos gastrointestinales en roedores de áreas urbanas con una mayor disponibilidad de alimento y densidad de personas, demostrando el alto riesgo de infección en lugares con un mayor crecimiento urbanístico (8).

Actualmente, durante exámenes de medicina preventiva en el Zoológico de Barranquilla, se ha identificado la presencia de parasitismos intestinales asociados principalmente a familias de nematodos (Strongyloidae, Trichinellidae, Ascarididae, Toxocaridae, Trychostrongyloidae, Ranidae, entre otros), protozoos (Eimeriidae, Entamoebidae, Balantidiidae y Sarcocystidae) y cestodos (Taeniidae e Hymenolepididae) en algunos animales de la colección (datos no publicados); no obstante, el papel de los roedores sinantrópicos en la trasmisión de estos parásitos permanece sin ser evaluado. Con base en lo anterior, el objetivo del presente estudio fue describir la prevalencia y caracterizar los parásitos gastrointestinales en roedores sinantrópicos capturados en el Zoológico de Barranquilla, Colombia, con el fin de explorar el papel epidemiológico de estos hospederos en la transmisión de dichas infecciones hacia los animales y humanos.

\section{MATERIALES Y MÉTODOS}

Descripción del área de estudio. El presente estudio se realizó en el Zoológico de Barranquilla (FUNDAZOO), ubicado en el departamento del Atlántico dentro del área urbana del municipio de Barranquilla (11000'39" N 74047'52" 0; 30 msnm), Colombia. Las condiciones ecológicas de esta zona se asemejan a las de un bosque seco tropical con una precipitación mensual entre 70 y $178 \mathrm{~mm}^{3}$ y una temperatura media de $29^{\circ} \mathrm{C}$ $\left(24-34^{\circ} \mathrm{C}\right)(10)$. 
Tipo de estudio y muestreo. Se realizó un estudio transversal, en el cual el universo de estudio fueron los roedores capturados durante el plan de control de roedores en las instalaciones del Zoológico de Barranquilla del 20 de junio a 09 de agosto del 2019. Para la captura de los roedores, se utilizaron trampas Tomahawk (medidas: $45 \mathrm{~cm}$ de largo x $13 \mathrm{~cm}$ de ancho y $13 \mathrm{~cm}$ de alto) con ayuda de cebos no tóxicos (mezcla de mazorca y mantequilla de maní) (2, $8)$, las cuales se ubicaron al azar en 55 puntos de captura de los sectores A, B, y C durante un total de 168 horas/trampa (Figura 1). Dicho tiempo fue distribuido en un período máximo de 42 horas semanales y 14 horas al día, por lo cual las trampas fueron colocadas tres veces por semana (lunes, miércoles y viernes) entre las 5:00 pm y 7:00 am del día siguiente, con una semana de descanso alternado. Previo a la realización del estudio, las trampas fueron colocadas durante un periodo de dos semanas con el fin de generar una adaptación de los animales a la presencia de estas.
Procesamiento de los roedores capturados. Luego de la captura, los animales eran trasladados vivos al área de necropsia para su manipulación según lo establecido en los estándares de bioseguridad y normas de procesamiento, acordes a los protocolos del centro de enfermedades infecciosas y prevención de Atlanta, Estados Unidos de América (11). Inicialmente, los roedores fueron anestesiados e insensibilizados en cámara anestésica, mediante inhalación de isoflurano al 5\% (Baxter, Cali, Colombia). Durante el período de sedación, por cada animal se determinó el sexo y el peso en gramos $(\mathrm{g}$ ) utilizando una balanza digital (Lexus, Nagoya, Japón). Para machos y hembras, la edad fue establecida según el peso vivo de los animales, mientras que la clasificación de las especies se realizó según los parámetros morfométricos: longitud total, largo de cola y longitud de las orejas (12).

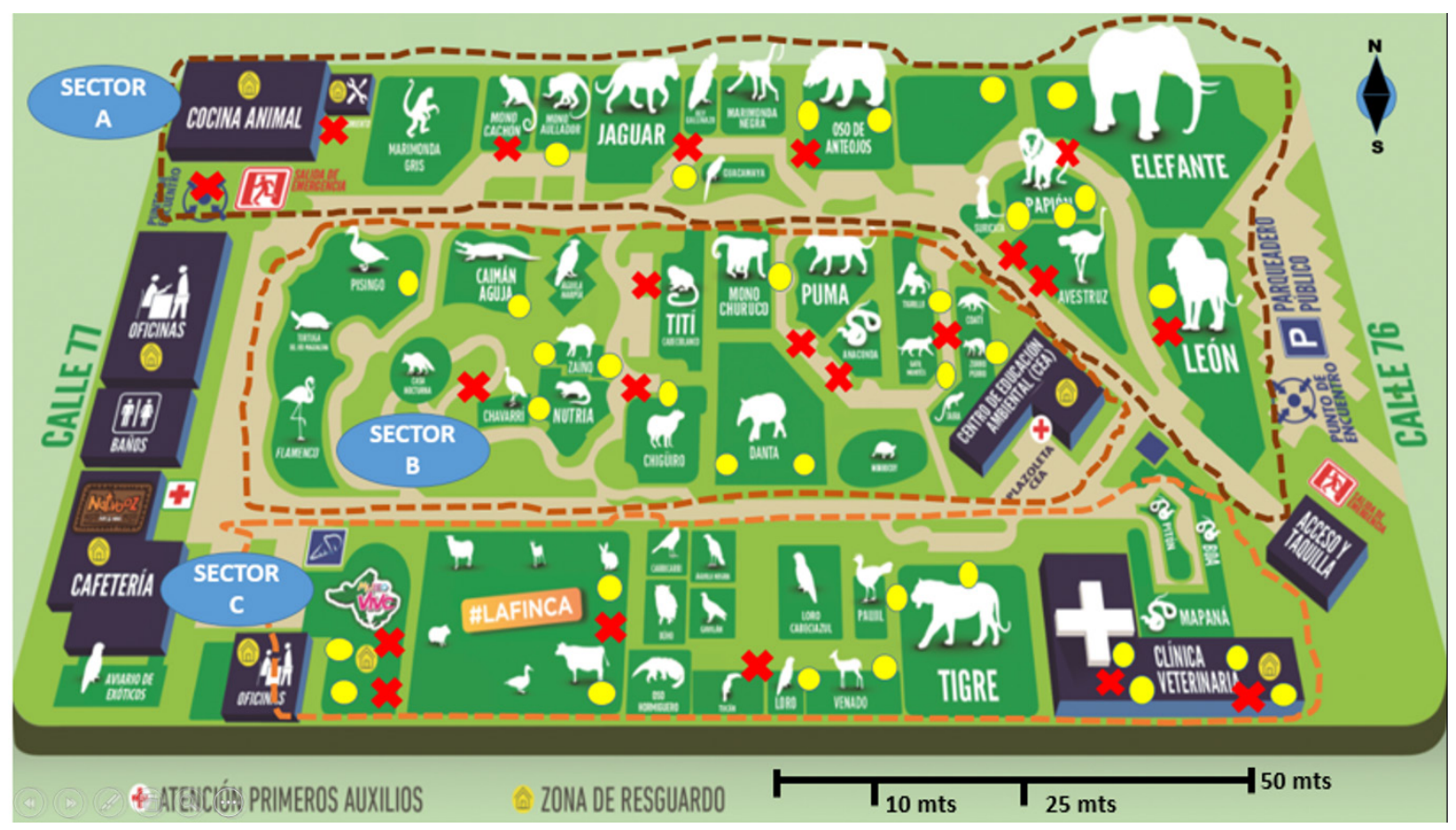

Figura 1.Distribución de los puntos de muestreo en el Zoológico de Barranquilla. Los círculos amarillos ( $\mathbf{J}$ ) y las $x$ rojas ( indican puntos de trampeo positivos y negativos a la captura de roedores. Las áreas de exhibición de los animales se encuentran en verde oscuro (_) y los senderos para los visitantes representados por el color beige ( ). La diversidad de especies en los diferentes sectores corresponde a: sector A (aves y mamíferos), sector B (aves, mamíferos y reptiles) y sector C (anfibios, aves, mamíferos y reptiles)

(Fuente: Tomado y modificado de FUNDAZOO https://www.zoobaq.org/info/mapa.php). 
Luego de obtener el plano de sedación e insensibilización, se realizó la eutanasia de los animales por sobredosis $(60 \mathrm{mg} / \mathrm{kg}) \mathrm{de}$ pentobarbital sódico intracardiaco (Euthanex, Bogotá, Colombia) (11). Al momento de la necropsia, se evaluó el tracto gastrointestinal en busca de endoparásitos adultos y lesiones macroscópicas compatibles con quistes parasitarios en hígado y peritoneo. Los helmintos de mayor tamaño fueron recolectados y preservados en formalina al $10 \%$ y trasladados al laboratorio clínico del zoológico para su identificación morfológica. Finalmente, un barrido del contenido intestinal presente en intestino delgado e intestino grueso fue realizado por cada animal y conservado en formalina al $10 \%$ hasta el diagnóstico parasitológico.

Diagnóstico parasitológico. Por cada barrido de contenido intestinal, las técnicas utilizadas para el diagnóstico de la infección e identificación de los parásitos gastrointestinales fueron el examen directo con solución salina fisiológica $(\mathrm{NaCl} 0.9 \%)$, flotación Sheather modificada y sedimentación Ritchie modificada $(13,14)$. Cada animal se consideró positivo a la infección con parásitos gastrointestinales cuando se observaron huevos o larvas en al menos una de las técnicas anteriores. La identificación morfológica de cada clase, familia y género de los parásitos adultos, como de sus formas inmaduras se realizó según las claves morfológicas descritas en literatura científica $(15,16,17,18)$.

Análisis de datos. La frecuencia de parásitos gastrointestinales se expresó en porcentajes, con su respectivo intervalo de confianza al 95\% (IC 95\%). Las diferencias entre las frecuencias de infección entre tipos de parásitos, edades y sector de captura de los roedores se exploraron mediante la prueba de chi-cuadrado $\left(X^{2}\right)$. El grado de concordancia entre las técnicas diagnósticas se determinó mediante un índice Kappa $(K)$. Todos los análisis fueron realizados en el programa SPSS v.23.0. Un valor $p<0.05$ fue considerado significativo. Todas las gráficas fueron realizadas en el programa GraphPad Prism v.8.1.1.

Consideraciones éticas. Todos los procedimientos se realizaron bajo los lineamientos de las buenas prácticas clínicas y de ética establecidas en el código sanitario para los animales terrestres (19). El aval de bioética fue otorgado por el subcomité de bioética de la Universidad Cooperativa de Colombia, seccional Bucaramanga, bajo los principios éticos para el manejo de los animales, establecidos en el Estatuto Nacional de protección para los animales, Ley 84 de 1989 y la resolución No. 008430 de 1993 del Ministerio de Salud (BogotáColombia).

\section{RESULTADOS}

Descripción del muestreo. Un total de 34 roedores (Rattus rattus) fueron capturados durante el período de estudio, de los cuales $53 \%$ (18/34) fueron clasificados como adultos y $47 \%$ (16/34) como sub-adultos. La mayoría de los animales fueron hembras $(61.8 \%, 21 / 34)$ con un peso promedio de $181.3 \mathrm{~g}$. Las frecuencias más altas de captura se observaron en los sectores B $(35.2 \%, 12 / 34)$ y C $(35.2 \%, 12 / 34)$, seguido por el sector A $(29.4 \%, 10 / 34)$.

Descripción de la frecuencia de infección por parásitos gastrointestinales. Del total de animales analizados, $82.4 \%$ (IC 95\% $=68.8 \%$ $95.8 \%, 28 / 34$ ) fueron positivos a la infección con parásitos gastrointestinales en al menos una prueba diagnóstica. Las mayores frecuencias de infección se observaron en hembras $(85.7 \%$, IC $95 \%=69.3 \%-102 \%, 18 / 21)$, adultos $(88.9 \%$, IC $95 \%=72.8 \%-104.9 \%, 16 / 18) \mathrm{y}$ animales del sector B $(91.7 \%$, IC $95 \%=73.3 \%$ $110 \%, 11 / 12)$. No se observaron diferencias significativas entre las categorías analizadas (sexo, edad y sector de captura) (Tabla 1).

Tabla 1. Descripción de la frecuencia de parásitos gastrointestinales identificadas en roedores $R$. rattus entre las diferentes categorías analizadas.

\begin{tabular}{|c|c|c|c|c|c|}
\hline Variable & $\mathbf{n}$ & Frecuencia & IC 95\% & $x^{2}$ & $\mathbf{p}$ \\
\hline \multicolumn{6}{|l|}{ Sexo } \\
\hline Hembra & 21 & $\begin{array}{c}85.7 \% \\
(18 / 21)\end{array}$ & $69.3-102 \%$ & & \\
\hline Macho & 13 & $\begin{array}{c}76.9 \% \\
(10 / 13)\end{array}$ & $50.4-103.4 \%$ & 041 & .838 \\
\hline
\end{tabular}

Edad

$\begin{array}{cccccc}\text { Adultos } 18 & \begin{array}{c}88.9 \% \\ (16 / 18) \\ 75 \%\end{array} & 72.8-104.9 \% & & \\ \text { Sub adultos } 16 & \begin{array}{c}75 \% \\ (12 / 16)\end{array} & 51.1-98.8 \% & & 0.740\end{array}$

Sector de captura

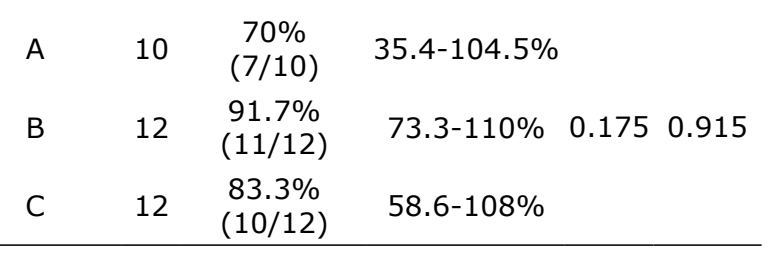


Respecto a la caracterización de las infecciones en las muestras positivas, los parásitos encontrados con mayor frecuencia fueron los nematodos $(63.2 \%$, IC $95 \%=49.2-77.2 \%, 31 / 49)$, seguido de los protozoos $(20.4 \%$, IC $95 \%=$ $8.7-32.1 \%, 10 / 49)$ y cestodos $(16.3 \%$, IC $95 \%$ $=5.6-27 \%, 8 / 49)$, con diferencias significativas entre los tres grupos $\left(x^{2}=20.7, p<0.01\right)$. Dentro de los nematodos, la familia encontrada con mayor frecuencia fue Strongylidae $(77.4 \%$, IC $95 \%=61.8-93 \%, 24 / 31)$, seguida de Ascarididae (12.9\%, IC 95\% $=0.4-25.4 \%, 4 / 31)$, Ancylostomatidae (3.2\%, IC 95\%=-3.3- 9.8\%, $1 / 31)$, Oxyuridae $(3.2 \%$, IC $95 \%=-3.3-9.8 \%$, $1 / 31)$ y Trichostrongylidae $(3.2 \%$, IC $95 \%=-3.3-$ $9.8 \%, 1 / 31)$, respectivamente. Para el caso de los protozoos, fueron las familias Eimeriidae $(60 \%$, IC $95 \%=23-96.9 \%, 6 / 10)$ y Entamoebidae (40\%, IC $95 \%=3-76.9 \%, 4 / 10)$, mientras que, para los cestodos, la única familia encontrada fue la Hymenolepididae $(100 \%$, IC $95 \%=100-100 \%$, 8/8) (Figura 2).

Al momento de la necropsia se observaron parásitos adultos en el 20.5\% (IC 95\% $=6.2$ $34.9 \%, 7 / 34$ ) de los animales analizados, de los cuales el $14.7 \%$ (IC $95 \%=2.1-27.2 \%, 5 / 34$ ) correspondieron a quistes hidatídicos formados por larvas inmaduras de Hymenolepis sp., y el $5.8 \%$ (IC $95 \%=-2.4-14.2 \%, 2 / 34$ ) a larvas de Strongylus sp., ubicadas en la luz del intestino.

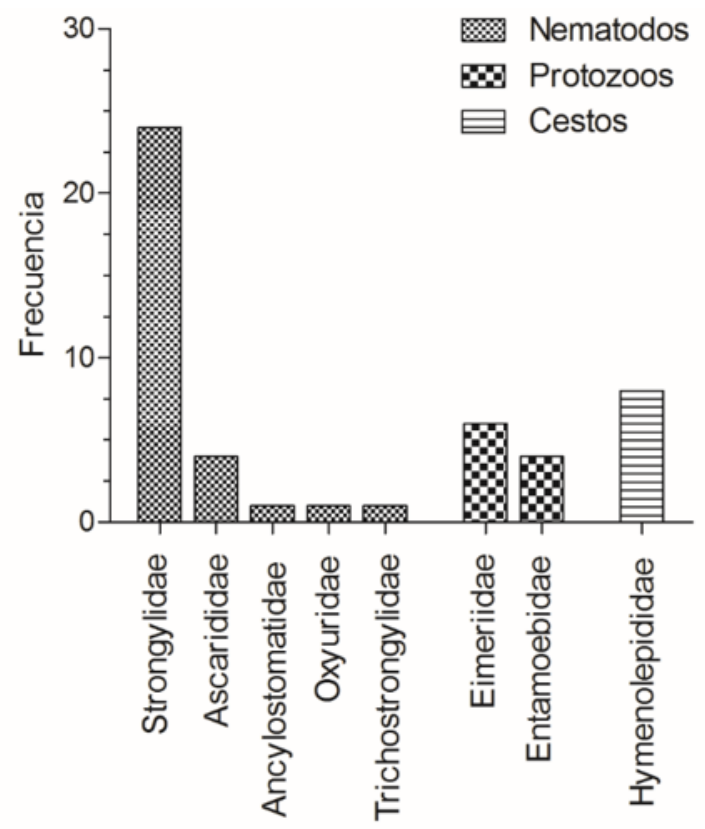

Figura 2. Frecuencia de las familias de parásitos gastrointestinales identificados en roedores sinantrópicos, capturados en el Zoológico de Barranquilla.
Dentro de las técnicas utilizadas, la técnica de sedimentación fue la más eficaz detectando una frecuencia de infección del $76.5 \%$ (IC 95\% = $61.4-91.4 \%, 26 / 34)$, seguido por la técnica directa $(67.6 \%$, IC $95 \%=51-84.2 \%, 23 / 34)$ y de flotación $(32.4 \%$, IC $95 \%=15.7-48.9 \%, 11 / 34)$. El mayor grado de concordancia, fue observado entre la técnica de sedimentación y directo con un $K=0.49$ (IC $95 \%=0.16$ a 0.83) (Tabla 2).

Tabla 2. Grado de concordancia entre pruebas coprológicas empleadas en roedores sinantrópicos, capturados en el zoológico de Barranquilla.

\begin{tabular}{ccc}
\hline Combinación & Índice Kappa (K) & IC 95\% \\
\hline $\begin{array}{c}\text { Sedimentación vs } \\
\text { Flotación }\end{array}$ & 0.26 & -0.02 a 0.54 \\
Sedimentación vs & 0.49 & 0.16 a 0.83 \\
Directo & 0.27 & -0.03 a 0.56 \\
Directo vs Flotación &
\end{tabular}

\section{DISCUSIÓN}

El Zoológico de Barranquilla es uno de los principales centros recreacionales de la región Caribe colombiana donde asisten alrededor de 300000 visitantes al año, y genera alrededor de 100 empleos directos e indirectos (20). En esta institución, los roedores sinantrópicos representan uno de los problemas de más difícil solución en el control de plagas, pues no solo provocan cuantiosas pérdidas económicas, si no también representan un riesgo de salud pública debido a su rol en la transmisión de enfermedades zoonóticas $(1,2,8,9)$.

En este estudio, se pudo observar la presencia exclusiva de roedores sinantrópicos $R$. rattus con una frecuencia de infección por parásitos gastrointestinales de $82.4 \%$. Estos resultados son similares a los reportados en centros urbanos de Perú y Cuba (64.1-77.4\%) $(1,2,21)$, pero considerablemente mayores a los observados en centros urbanos y rurales de México, en donde la prevalencia en estos mismos hospederos osciló entre el 42.7 y $52.2 \%(22,23)$. Considerando que, en América, la frecuencia de infección por parásitos gastrointestinales en roedores sinantrópicos provenientes de zoológicos, ha sido poco estudiada, la alta frecuencia de infección aquí observada puede ser explicada debido a la mayor densidad de hospederos definitivos e intermediaros presentes en el zoológico. En Brasil, algunos estudios realizados en carnívoros $(24,25)$, llegaron a la conclusión que las 
condiciones de cautiverio pueden incrementar la prevalencia de infección por parásitos gastrointestinales, favoreciendo la diseminación hacia otros animales, inclusive el humano. En este sentido, mantener los programas de control de parásitos gastrointestinales, e intensificar el control de roedores, es importante para evitar la diseminación de estos parásitos a otras especies. Respecto a la presencia exclusiva de $R$. rattus (una especie arborícola) y su captura con trampas de piso, puede ser un indicador del nicho ecológico adecuado para esta especie, en donde la alta disponibilidad de alimento, los lugares de refugio, la falta de predadores naturales, y la capacidad de adaptación a las estrategias de control, puede generar una ventaja biológica frente a las demás especies (26). No obstante, el efecto del tipo de trampa empleada en el presente estudio la cual disminuye la probabilidad de captura de otras especies sinantrópicas $(2,8)$, y el efecto de las estrategias de control de roedores en el zoológico, las cuales están enfocadas en roedores terrestres, también pueden explicar este resultado.

En cuanto a la caracterización de parásitos gastrointestinales, se observó que los nematodos fueron el grupo más prevalente, seguido por los protozoos y cestodos. Estos resultados son similares a los reportados en los animales de la colección del zoológico (datos no publicados), sugiriendo el papel de estos roedores como posibles reservorios en el área de estudio $(1,2,8,9)$. Para el caso de los nematodos, la familia Strongylidae presentó una mayor frecuencia de infección, estos resultados son similares a los reportados en $R$. rattus, $R$. norvegicus y $M$. musculus de la isla de Cuba (21). Sin embargo, difieren de los observados en $R$. rattus y $R$. norvegicus del área metropolitana de Lima, Perú (1), en donde los nematodos identificados con mayor frecuencia pertenecieron a la familia Gongylonematidae. Esto indica que la prevalencia de cada familia varía dependiendo las condiciones ecológicas de la zona y de la diversidad de hospederos $(26,27)$. La alta presencia de parásitos de la familia Strongylidae, puede estar asociada a las diversas vías de infección entre las que se incluye: la vía fecooral, percutánea, y transmamaria (28), así como a una posible adaptación de estos parásitos al sistema inmune de $R$. rattus (28). Este escenario, aunque es común en áreas urbanas, representa un riesgo de infección para las personas y animales en el zoológico, al considerarse una familia de interés zoonótico (2). Estos resultados motivaron intervenciones en el programa de control de roedores en el zoológico con el fin de reducir el riesgo de infección hacia los humanos.

La alta proporción de la familia Hymenolepididae dentro de los cestodos identificados en $R$. rattus, concuerdan con lo observado en otros países de América, en donde las especies $R$. norvegicus, $M$. musculus y Sigmodon hirsutus, también se han encontrado infectados con dicha familia $(1,2,8,9)$. Aunque la transmisión directa e indirecta de estos parásitos es común entre animales silvestres, indicando la posible infección hacia animales de la colección, la importancia zoonótica de estos es baja, puesto que la infección hacía el humano solo se produce mediante el consumo accidental de hospederos intermediarios como escarabajos y pulgas de los géneros: Tenebrio, Tribolium, Ctenocephalides, Pulex y Xenopsilla, infectados con el cisticercoide (9). De otro lado, las altas prevalencia de protozoos de las familias Eimeriidae y Entamoebidae, son mayores a las observadas en países de Centro América en los cuales la prevalencia de estas familias no supera el $5 \%$ (21). Teniendo en cuenta que el consumo de agua contaminada con quistes de estos parásitos es considerado la principal vía de infección en animales y humanos (21), es importante que futuros estudios determinen si el agua utilizada en el zoológico es una fuente de infección.

Finalmente, aunque en el presente estudio empleando técnicas parasitológicas se determinó una alta frecuencia de infección por parásitos gastrointestinales, así como la presencia de algunas familias de importancia zoonótica, la falta de técnicas altamente sensibles y específicas dificultó conocer en detalle el papel de estos roedores en la trasmisión de especies de importancia en la salud pública y animal. Futuros estudios empleando técnicas diagnósticas como la PCR, PCR en tiempo real, entre otras técnicas moleculares, son necesarios para solucionar estas dificultades. En conclusión, la alta prevalencia de infección por parásitos gastrointestinales en $R$. rattus del Zoológico de Barranquilla evidencia la importancia de estos animales como fuentes de infección para otros animales y los seres humanos. La alta frecuencia de parásitos de la familia Strongyloidae en los roedores capturados en el sitio de estudio, sugiere un riesgo de salud pública, lo cual motivó a intensificar las medidas de control para disminuir el riesgo de infección y mejorar el estatus sanitario. 


\section{Conflicto de intereses}

Los autores declararon que no existen conflictos de interés potenciales con respecto a la investigación, autoría o publicación de este artículo.

\section{Agradecimiento}

El presente trabajo fue realizado gracias a la cooperación científica del clúster establecido entre la Fundación Botánica y Zoológica de Barranquilla (FUNDAZOO) y la Universidad Cooperativa de Colombia (UCC), sede Bucaramanga. Agradecemos a FUNDAZOO por proveer los insumos, equipos, instalaciones y el apoyo financiero al estudiante durante la realización del estudio.

\section{REFERENCIAS}

1. De Sotomayor $R$, Serrano $E$, Tantaleán M, Quispe M, Casas G. Identificación de parásitos gastrointestinales en ratas de Lima metropolitana. Rev Inv Vet Perú. 2015; 26(2):273-281. https://doi.org/10.15381/ rivep.v26i2.11003

2. Abad D, Chávez A, Pinedo R, Tantaleán $M$, Gonzáles O. Helmintofauna gastrointestinal de importancia zoonótica y sus aspectos patológicos en roedores (Rattus spp) en tres medioambientes. Rev Inv Vet Perú. 2016; 27(4):736-750. https://doi.org/10.15381/ rivep.v27i4.12568

3. Torres-Castro MA. ¿Son los roedores sinantrópicos una amenaza para la salud pública de Yucatán? Rev Biomed. 2017; 28(3):183-190. https://doi.org/10.32776/ revbiomed.v28i3.566

4. Londoño $A F$, Acevedo-Gutiérrez $L Y$, Marín D, Contreras V, Díaz FJ, Valbuena G. Wild and domestic animals likely involved in rickettsial endemic zones of Northwestern Colombia. Ticks Tick Borne Dis. 2017; 8(6):887-894. https://doi.org/10.1016/j. ttbdis.2017.07.007

5. Ospina C, Rincó M, Soler D, Hernández P. The role of rodents in the transmission of Leptospira spp. in swine farms. Rev. Salud Pública. 2017; 19(4):555-561. https://doi. org/10.15446/rsap.v19n4.41626

6. Quintero J, Londoño A, Díaz F, Agudelo P, Arboleda M, Rodas J. Ecoepidemiología de la infección por rickettsias en roedores, ectoparásitos y humanos en el noroeste de Antioquia, Colombia. Biomedica. 2013; 33(1):38-51. https://doi.org/10.7705/ biomedica.v33i0.735
7. Rodriguez E, Cantillo O, Prieto A, Cucunuba Z. Heterogeneity of Trypanosoma cruzi infection rates in vectors and animal reservoirs in Colombia: a systematic review and meta-analysis. Rev. Parasites and vectors. 2019; 12(308):1-19. https://doi. org/10.1186/s13071-019-3541-5

8. Duque BA, Aranzazu D, Agudelo-Flórez P, Londoño AF, Quiroz V, Rodas JD. Rattus norvegicus como indicador de la circulación de Capillaria hepatica y Taenia taeniaeformis en la plaza minorista de Medellín, Colombia. Biomedica. 2012; 32(4):510-518. https:// doi.org/10.7705/biomedica.v32i4.442

9. Sepulveda M, Pardo M. Hallazgo de Cestodos de la familia Hymenolipidae en el ratón algodonero del Sur (Sigmodon hirsutus) en Huila, Colombia. Rev Med Vet Zoot. 2014; 61(1):11-16. https://doi.org/10.15446/ rfmvZ.v61n1.43879

10. Aldana-Domínguez J, Montes C, Martínez M, Medina N, Hahn J, Duque $M$. Biodiversity and ecosystem services knowledge in the Colombian Caribbean: progress and challenges. Trop. Conserv. Sci. 2017; 10:1-41. https://doi. org/10.1177/1940082917714229

11. Carro F, Pérez D, Lamosa A, Schmalenberger $H$, Pardavila X, Gegúndez I, Soriguer R. Eficiencia de tres tipos de trampas para la captura de micromamíferos. Galemys. 2007; 19( $n^{\circ}$ especial):73-81. http://www. secem.es/wp-content/uploads/2013/03/ Galemys-19-NE-07-Carro-73-81.pdf

12. William GB, Taylor J. Body Mass, Testes Mass, and Sperm Size in Murine Rodents, J Mammal. 2000; 81(3):758768. https://doi.org/10.1644/15451542(2000)081<0758:BMTMAS >2.3.CO;2 
13. Benavides O. Técnicas para el diagnóstico de endoparásitos de importancia veterinaria. Primera Edición. Bogotá, Colombia: Universidad de la Salle; 2013. https:// sibbila.lasalle.edu.co/janium-bin/detalle. pl?Id $=20200306141546$

14. Silva RA, Tonani KA, Fregonesi BM, Mariano AP, Ferrassino MD, Trevilato TM. Adaptation of Ritchie's Method for Parasites Diagnosing with Minimization of Chemical Products. Interdiscip Perspect Infect Dis. 2012; 2012(1):1-5. https://doi. org/10.1155/2012/409757

15. Sepulveda MS, Kinsella JM. Helminth collection and identification from wildlife. J Vis Exp. 2013; 14(82):1-5. https://doi. org/10.3791/51000

16. Kia E, Shahryary-Rad E, Mohebali M, Mahmoudi M, Mobedi I, Zahabiun F. Endoparasites of Rodents and Their Zoonotic Importance in Germi, Dashte-Mogan, Ardabil Province, Iran. Iran J Parasitol. 2010; 5(4):15-20. http://ijpa.tums.ac.ir/ index.php/ijpa/article/view/152

17. Zajac AM, Gary CA. Veterinary Clinical Parasitology. Hoboken, NJ: Wiley-Blackwell; 2011. https://www.wiley.com/en-us/Veteri nary +Clinical+Parasitology $\% 2 \mathrm{C}+8$ th+Editi on-p-9780813820538

18. Van Wyk J, Mayhew E. Morphological identification of parasitic nematode infective larvae of small ruminants and cattle: A practical lab guide. J Vet Res. 2013; 80(1):a539. https://doi.org/10.4102/ojvr. v80i1.539

19. Thiermann AB. International standards: the World Organization for Animal Health Terrestrial Animal Health Code. Rev Sci Tech. 2015; 34(1):277-81. https://doi. org/10.20506/rst.34.1.2340

20. Vargas J, Máttar S, Monsalve S. Bacterias patógenas con alta resistencia a antibióticos: estudio sobre reservorios bacterianos en animales cautivos en el zoológico de Barranquilla. Infect. 2010; 14(1). http:// www.revistainfectio.org/index.php/infectio/ article/view/32

21. Companioni A, Atencio I, Cantillo J, Hernández N, González A, Núñez, F. Prevalence of endoparasites in synanthropic rodents (Rodentia: Muridae) in an area of Havana, Cuba. Rev Cubana Med Trop. 2016; 68(3):240-247. http://revmedtropical.sld. cu/index.php/medtropical/article/view/142
22. Panti-May JA, Hernández-Betancourt SF, Rodríguez-Vivas RI, Robles MR. Infection levels of intestinal helminths in two commensal rodent species from rural households in Yucatan, Mexico. J Helminthol. 2015; 89(1):42-48. https:// doi.org/10.1017/S0022149X13000576

23. Panti-May JA, Palomo-Arjona E, GurubelGonzález Y, Torres-Castro MA, Vidal-Martínez, VM, Machain-Williams C, Hernándezbetancour F, del Rosario-robles M. New host, geographical records, and factors affecting the prevalence of helminths infection from synanthropic rodents in Yucatán, Mexico. Helminthologia. 2017; 54(3):231-239. https://doi.org/10.1515/helm-2017-0030

24. Müller GC, Greinert JA, Silva Filho $\mathrm{HH}$. Frequency of intestinal parasites in felines kept in zoos. Arq Bras Med Vet Zootec. 2005; 57(4):559-561. https://doi.org/10.1590/ S0102-09352005000400021

25. Figueiroa $M$, Bianque J, Dowell $M$, Alves $R$, Evêncio A. Perfil coproparasitológico de mamíferos silvestres en cautiverio en el estado de Pernambuco, Brasil. Bol Chil Parasitol. 2001; 25:3-4. http://dx.doi. org/10.4067/S0716-07202001000300009

26. Cantillo J, Hernández N, Companioni A, Berovides V, Anaya J. Dinámica poblacional de múridos (ratas) en dos localidades de Ciudad la Habana. Rev Electrón Vet. 2011; 12(11):1-10. https://www.redalyc.org/ pdf/636/63622049013.pdf

27. Iannacone J, Alvariño L. Helmintofauna de Rattus rattus (Linnaeus, 1758) y Rattus norvegicus (Berkenhout, 1769) (Rodentia: Muridae) en el distrito de San Juan de Lurigancho, Lima - Perú. Rev Perú Med Exp Salud pública. 2002; 19(3): 136-141. https://rpmesp.ins.gob.pe/index.php/ rpmesp/article/view/819

28. Viney M. Strongyloides. Parasitology. 2017; 144(3):259-262. https://doi.org/10.1017/ $\underline{\mathrm{S} 0031182016001773}$ 